\title{
Avaliação epidemiológica do mormo no Brasil
}

\author{
Epidemiological evaluation of glanders in Brazil \\ Evaluación epidemiológica del muermo en Brasil
}

\section{Resumo}

Esta pesquisa teve por objetivo analisar o perfil epidemiológico do mormo no Brasil, no período de 2010 a 2019 . Foi realizado um estudo transversal, descritivo, retrospectivo e qualiquantitativo que utilizou como unidades de análise todos os estados brasileiros. A amostra foi delimitada ao período de 2010 a 2019, e os dados referentes aos casos notificados de Mormo foram coletados a partir das informações disponíveis em banco de dados oficiais, sendo elas, ano, semestre, unidade da federação, número total de casos e número total de focos. No período foram notificados 1398 casos de Mormo no Brasil. Acre e Amapá não relataram ocorrência da doença, enquanto Pernambuco, seguido por Mato Grosso e São Paulo lideraram em número absoluto de casos. Os estados que apresentaram maior prevalência foram Amazonas (2,59/1000 cab.), Rio Grande do Norte (2,10/1000 cab.) e Distrito Federal (1,94/1000 cab.). A região Norte apresentou menor concentração de casos por foco $(1,45)$, enquanto a região sudeste demonstrou maior concentração dos casos $(2,73)$ por foco. Conclui-se que o Brasil possui baixa prevalência de mormo, com concentração maior de casos e focos na região Nordeste, e maior prevalência no estado do Amazonas. Devido a importância do mormo para a saúde pública, medidas de vigilância ativa da doença nos animais e ações de prevenção, no âmbito de Saúde Única, devem ser executadas.

Palavras-chave: Burkholderia mallei; Equídeos; Notificação compulsória.

\section{Abstract}

This study was developed to analyze the epidemiological profile of glanders in Brazil from 2010 to 2019 . A crosssectional, descriptive, retrospective and qualitative-quantitative study was carried out using all Brazilian states as units 
of analysis. The sample was delimited to the period from 2010 to 2019, and data referring to notified cases of glanders were collected from information available in the official database, namely, year, semester, federation unit, total number of cases and total number of outbreaks. In the period, 1398 cases of the disease were reported in Brazil. Acre and Amapá did not report its occurrence, whereas Pernambuco, followed by Mato Grosso and São Paulo, led the absolute number of cases. The states with the highest prevalence were Amazonas (2.59/1000 head), Rio Grande do Norte (2.10/1000 head) and the Federal District (1.94/1000 head). The north region showed the lowest concentration of cases per focus (1.45), and the southeast region the highest (2.73). In conclusion, Brazil has a low prevalence of glanders, with a higher concentration of cases and outbreaks occurring in the northeast region and a higher prevalence in the state of Amazonas. Due to the importance of glanders for public health, measures of active surveillance of the disease in animals and prevention actions, in the One Health context, must be implemented.

Keywords: Burkholderia mallei; Compulsory notification; Equids.

\section{Resumen}

Esta investigación tuvo como objetivo analizar el perfil epidemiológico del muermo en Brasil, de 2010 a 2019. Se realizó un estudio transversal, descriptivo, retrospectivo y cualiquantitativo utilizando todos los estados brasileños como unidades de análisis. La muestra se delimitó al período de 2010 a 2019, y los datos referentes a los casos notificados de Mormo se recolectaron a partir de la información disponible en la base de datos oficial, es decir, año, semestre, unidad federativa, número total de casos y número total de brotes. En el período se notificaron 1398 casos de Mormo en Brasil. Acre y Amapá no reportaron la ocurrencia de la enfermedad, mientras que Pernambuco, seguido por Mato Grosso y São Paulo lideraron el número absoluto de casos. Los estados con mayor prevalencia fueron Amazonas (2,59 / 1000 cab.), Rio Grande do Norte (2,10 / 1000 cab.) Y el Distrito Federal (1,94 / 1000 cab.). La región Norte mostró una menor concentración de casos por foco $(1,45)$, mientras que la región Sudeste mostró una mayor concentración de casos $(2,73)$ por foco. Se concluye que Brasil tiene una baja prevalencia de muermo, con una mayor concentración de casos y brotes en la región Nordeste, y una mayor prevalencia en el estado de Amazonas. Debido a la importancia del muermo para la salud pública, se deben llevar a cabo medidas de vigilancia activa de enfermedades en animales y acciones de prevención, en el contexto de Sanidad Única.

Palabras clave: Burkholderia mallei; Équidos; Notificación obligatoria.

\section{Introdução}

O mormo é uma doença zoonótica altamente contagiosa para os equídeos, e frequentemente fatal ao ser humano. Ela é causada por uma bactéria, a Burkholderia mallei cujos equídeos são altamente susceptíveis sendo considerados reservatórios naturais para a infecção (Neubauer et al., 2005; Dittmann et al., 2015; Mota \& Ribeiro, 2016).

No Brasil, a equinocultura tem se destacado, e em concordância com dados do Ministério da Agricultura, Pecuária e Abastecimento (MAPA) a expansão da exportação de cavalos vivos cresceu significativamente entre 1997 e 2009 , alcançando $524 \%$, além disso, o país é um dos principais exportadores de carne equina (Saind, et al., 2016).

Os equídeos se infectam por meio do consumo de água e alimentos contaminados, sendo a via oral a principal porta de entrada do agente, mas a infecção pode ocorrer por via aerógena, ou seja, pela inalação da bactéria ou também por via cutânea, a partir de feridas na pele provocadas por objetos contaminados. Em seres humanos, o contágio se dá por meio do contato direto com animais infectados, inalação de aerossóis e feridas que tenham contato com secreção de animais positivos (Falcao, Silva, Mota, 2019).

A doença não apresenta predileção quanto a idade e sexo dos equídeos acometidos, porém animais expostos a condições estressantes, má alimentação e locais contaminados estão mais sujeitos a contrair a doença. Por isso, propriedades que utilizam os equídeos como animais de tração, atividade está bastante estressante, os prejuízos econômicos são consideráveis, principalmente pela morte dos animais e pela manutenção de animais muitas vezes debilitados e impróprios ao trabalho (Mota et al., 2000).

Mesmo a doença sendo zoonótica, a maioria da população desconhece a doença, o que coloca em risco sua saúde quando estão em contato com animais doentes (Falcao, Silva, Mota, 2019).

A sintomatologia frequentemente descrita nos casos de mormo é febre, tosse e corrimento nasal. Quadros de septicemia podem ocorrer na fase aguda da doença, levando o animal a morte. Já na fase crônica, a doença pode apresentar três formas de manifestações clínicas: nasal (presença de lesões nodulares em mucosa nasal), pulmonar (pneumonia crônica, tosse, dispneia, 
febre, apatia e caquexia), e cutânea (presença de nódulos ao longo do trajeto dos vasos linfáticos) (Galyov et al., 2010).

Nem todos os equídeos apresentam sinais clínicos da doença. Desta forma, os principais responsáveis pela disseminação da doença são os indivíduos assintomáticos (Rosado, 2018). Este fato é preocupante, principalmente aliado a falta de conhecimento dos proprietários de equídeos com relação aos aspectos epidemiológicos e clínicos da doença.

No Brasil, o Programa Nacional de Sanidade dos Equídeos (PNSE) propõe ações de Vigilância e Defesa Sanitária Animal. A Instrução Normativa n ${ }^{\circ}$ 6, de 16 de janeiro de 2018 regula as normas para erradicação e controle do mormo no Brasil, tendo como base ações relacionadas a interdição e saneamento de propriedades com animais positivos (Brasil, 2008; Vargas et al., 2015; Brasil, 2018).

O mormo não tem cura, por isso, animais positivos deverão ser sacrificados e incinerados ou enterrados no próprio local, assim como todos os materiais utilizados nas instalações que possam estar contaminados (Brasil, 2018).

Como medidas de controle da doença, preconiza-se a desinfecção das instalações e fômites, desinfecção de equipamentos (cabrestos, arreios), abolição de cochos coletivos, uso de equipamentos de proteção individual (EPI) pelos tratadores e manipuladores dos equídeos, controle de trânsito interestadual (apresentação de exame negativo de mormo), e notificação de foco suspeito (Brasil, 2018; Rosado, 2018). Neste contexto, a presente pesquisa tem por objetivo analisar o perfil epidemiológico do mormo no Brasil, no período de 2010 a 2019, visando enfatizar a importância das medidas de controle e prevenção da doença.

\section{Metodologia}

Para esta pesquisa foi realizado um estudo transversal, descritivo, retrospectivo e qualiquantitativo (Pereira et al. 2018) que utilizou como unidades de análise todos os estados brasileiros.

A amostra foi delimitada ao período de 2010 a 2019, e os dados referentes aos casos notificados de Mormo foram coletados a partir das informações disponíveis em banco de dados oficiais da Organização Internacional de Epizootias (OIE) e do Ministério da Agricultura, Pecuária e Abastecimento (MAPA).

As informações obtidas foram: ano, semestre, unidade da federação, número total de casos e número total de focos. Dados referentes ao rebanho susceptível foram coletados do Instituto Brasileiro de Geografia e Estatística (IBGE), no painel Pesquisa da Pecuária Municipal, para cálculo das taxas de prevalência da doença.

Para o cálculo da prevalência da doença, utilizou-se a seguinte equação matemática:

$$
\text { Coef. Prevalência }=\frac{\text { número casos notificados }}{\text { rebanho estimado }} \times 1.000
$$

Após coleta, os dados foram analisados por meio de estatística descritiva simples e os resultados apresentados em formato de gráficos e tabelas.

\section{Resultados e Discussão}

De acordo com os dados obtidos, o efetivo de rebanho equino no Brasil de 2010 a 2019, por unidade da federação, está descrito na Tabela 1. 
Tabela 1 - Efetivo de rebanho equino no Brasil, de acordo com as unidades da federação, de 2010 a 2019.

\begin{tabular}{|c|c|c|c|c|c|c|c|c|c|c|}
\hline & 2010 & 2011 & 2012 & 2013 & 2014 & 2015 & 2016 & 2017 & 2018 & 2019 \\
\hline AC & 67952 & 68114 & 65301 & 64871 & 76061 & 81699 & 97999 & 94821 & 95229 & 93315 \\
\hline $\mathbf{A L}$ & 56310 & 57982 & 58122 & 58228 & 60414 & 64126 & 65045 & 74573 & 85966 & 92025 \\
\hline AP & 5294 & 5683 & 5436 & 5566 & 5790 & 8948 & 8207 & 10068 & 10432 & 10179 \\
\hline AM & 12803 & 13685 & 15678 & 15479 & 22284 & 25363 & 27758 & 27251 & 28019 & 28032 \\
\hline BA & 586643 & 555905 & 518051 & 485356 & 470761 & 459727 & 481869 & 495373 & 508892 & 505568 \\
\hline CE & 138346 & 138720 & 136071 & 128602 & 131851 & 135046 & 138808 & 117110 & 117062 & 121110 \\
\hline DF & 7270 & 7200 & 12000 & 17162 & 18695 & 20320 & 21080 & 19442 & 18892 & 17929 \\
\hline ES & 71411 & 73489 & 74916 & 74585 & 76626 & 72927 & 66451 & 57768 & 54755 & 56902 \\
\hline GO & 428367 & 426950 & 401381 & 394799 & 385525 & 380405 & 365611 & 364174 & 366468 & 381095 \\
\hline MA & 170347 & 173739 & 173926 & 175575 & 176718 & 185722 & 197529 & 220220 & 236618 & 228496 \\
\hline MT & 344918 & 350001 & 319294 & 245153 & 302543 & 418766 & 372028 & 380277 & 425553 & 441626 \\
\hline MS & 344589 & 339136 & 338093 & 337124 & 337185 & 339781 & 354797 & 278482 & 330044 & 361005 \\
\hline MG & 802049 & 787129 & 785277 & 758880 & 763780 & 774639 & 762006 & 811337 & 874513 & 846197 \\
\hline PA & 289261 & 297082 & 270658 & 284437 & 312077 & 342779 & 380845 & 351806 & 429310 & 445603 \\
\hline PB & 48073 & 48284 & 44758 & 46262 & 49975 & 52683 & 54461 & 55965 & 59536 & 62305 \\
\hline PR & 341481 & 331689 & 325837 & 312626 & 301931 & 300781 & 296488 & 280717 & 282448 & 281139 \\
\hline PE & 139811 & 139111 & 123843 & 128027 & 125994 & 131567 & 124806 & 126358 & 132642 & 125968 \\
\hline $\mathbf{P I}$ & 112423 & 109936 & 106538 & 102092 & 99679 & 95990 & 93942 & 91509 & 69004 & 66816 \\
\hline RJ & 106248 & 115178 & 120572 & 113203 & 116473 & 100220 & 98884 & 100334 & 100231 & 101998 \\
\hline RN & 43112 & 45100 & 44454 & 53552 & 62654 & 63816 & 64585 & 64555 & 63658 & 67444 \\
\hline RS & 470199 & 472778 & 468691 & 535299 & 540815 & 535227 & 537159 & 553191 & 527868 & 520760 \\
\hline Ro & 161050 & 169585 & 175484 & 173440 & 166507 & 166259 & 164607 & 166722 & 184611 & 198436 \\
\hline RR & 28170 & 30276 & 28349 & 28548 & 29502 & 30399 & 27045 & 37063 & 37360 & 35020 \\
\hline SC & 114372 & 120331 & 122565 & 118342 & 120001 & 128676 & 140934 & 112766 & 105448 & 132210 \\
\hline SP & 379379 & 368833 & 358807 & 347411 & 363380 & 365068 & 365117 & 355554 & 343903 & 348124 \\
\hline SE & 72830 & 73712 & 73385 & 67922 & 68425 & 69567 & 76678 & 69245 & 66022 & 66478 \\
\hline TO & 171545 & 190973 & 195698 & 239535 & 264955 & 200787 & 191397 & 195328 & 196778 & 214374 \\
\hline
\end{tabular}

Fonte: IBGE (2021).

Os estados que apresentaram efetivo médio de rebanho equino maior durante o período de estudo, foram Minas Gerais, Rio Grande do Sul e Bahia.

O Brasil possui o terceiro maior rebanho equino do mundo e o maior da América Latina. A atividade envolve vários segmentos, dentre eles, a produção de insumos, a criação e a destinação final, o que movimenta $\mathrm{R} \$ 7,3$ bilhões por ano e gera cerca de 3,2 milhões de empregos diretos e indiretos (Said, Nardi Junior, Domingues, 2016).

O efetivo de rebanho equino significativo que o Brasil possui aliado a falhas de manejos favorecem a ocorrência de doenças infecciosas, dentre elas, o mormo, que é uma afecção que possui medidas preventivas previstas no Regulamento de Defesa Sanitária Animal do MAPA.

De 2010 a 2019 foram notificados 1.398 casos de mormo no Brasil. A distribuição anual dos casos está descrita na Figura 1. 
Figura 1 - Distribuição anual dos casos de Mormo notificados no Brasil, 2010 a 2019.

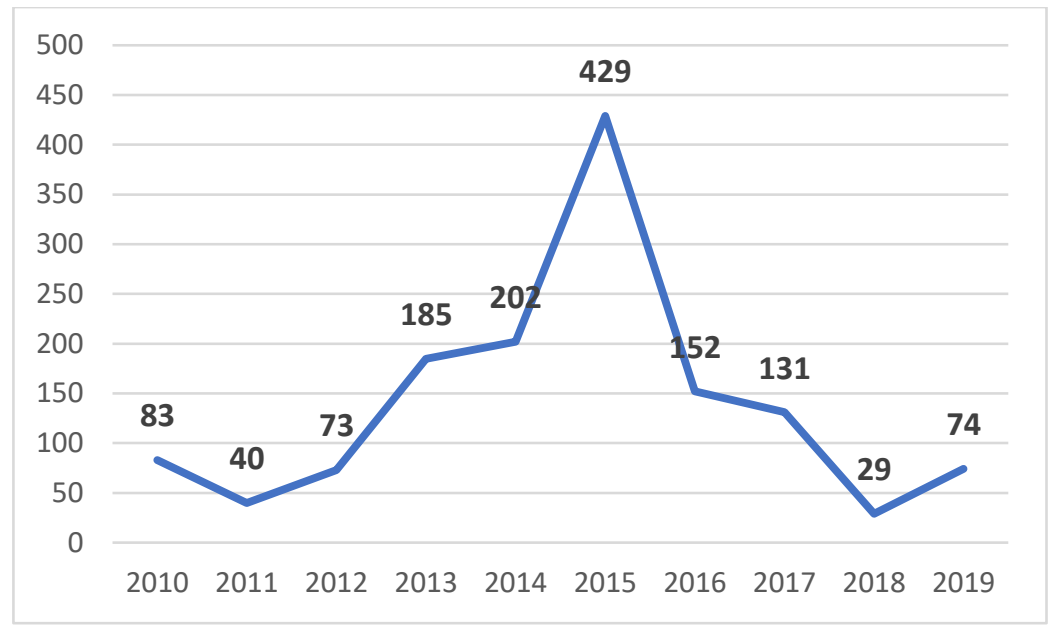

Fonte: Autores.

Ao analisar a distribuição anual dos casos notificados nota-se que a partir de 2011 houve um aumento acentuado do número de casos até o ano de 2015, ano este de maior ocorrência. Oliveira (2016) afirmaram, que em 2015, 19 estados brasileiros notificaram casos de mormo.

A partir de 2016, o número de casos notificados caiu drasticamente, o que pode indicar eficiência das medidas preventivas, pois muitos estados passaram a exigir o exame negativo para o mormo para trânsito de equídeos.

Em 1960, após relatos da ocorrência da doença, a mesma parecia ter sido erradicada no Brasil, porém, em 1999, o mormo ressurgiu nos estados de Alagoas e Pernambuco, e desde então relata-se a ocorrência de casos em vários estados brasileiros (Mota et al., 2000).

A distribuição dos casos notificados de mormo por região brasileira, de acordo com o MAPA, está descrita na Figura 2.

Figura 2 - Casos de Mormo notificados no Brasil de 2010 a 2019 de acordo com a região.

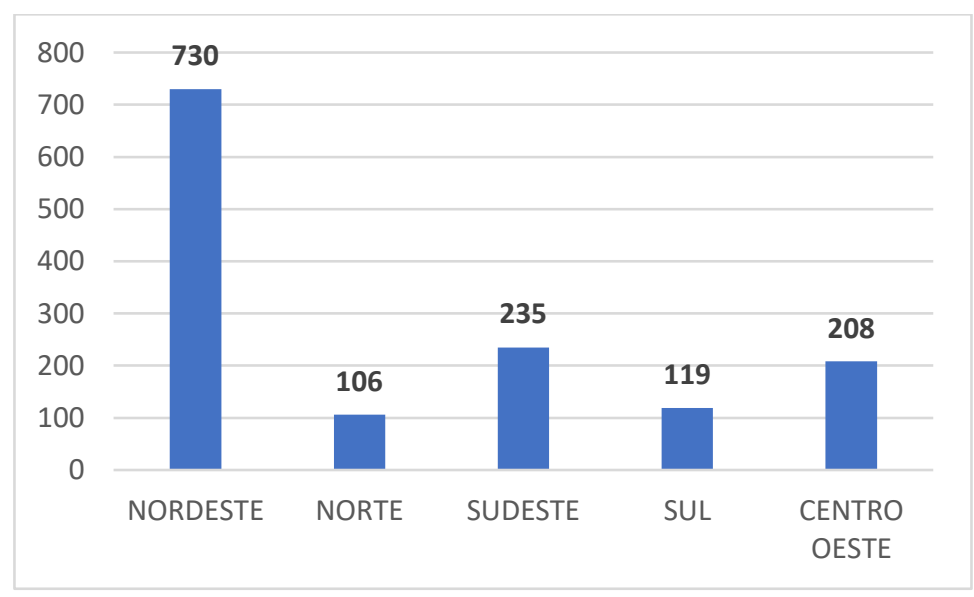

Fonte: Autores.

A maioria dos casos notificados no período de estudo ocorreram na região Nordeste do Brasil. De acordo com a Tabela 1, a região Nordeste possui o segundo maior rebanho de equinos do Brasil, ficando atrás apenas do estado de São Paulo. Mesmo assim, a região apresentou três vezes mais número de casos notificados. Este fato pode estar relacionado com a presença maciça ainda nesta região do uso dos equídeos como meio de transporte e trabalho, aliado a falhas de manejo sanitário, o que torna os animais mais propensos ao contágio. 
A distribuição por estado da federação, de acordo com o MAPA, está descrita na Figura 3.

Figura 3 - Casos de Mormo notificados no Brasil de 2010 a 2019 de acordo com a unidade da federação.

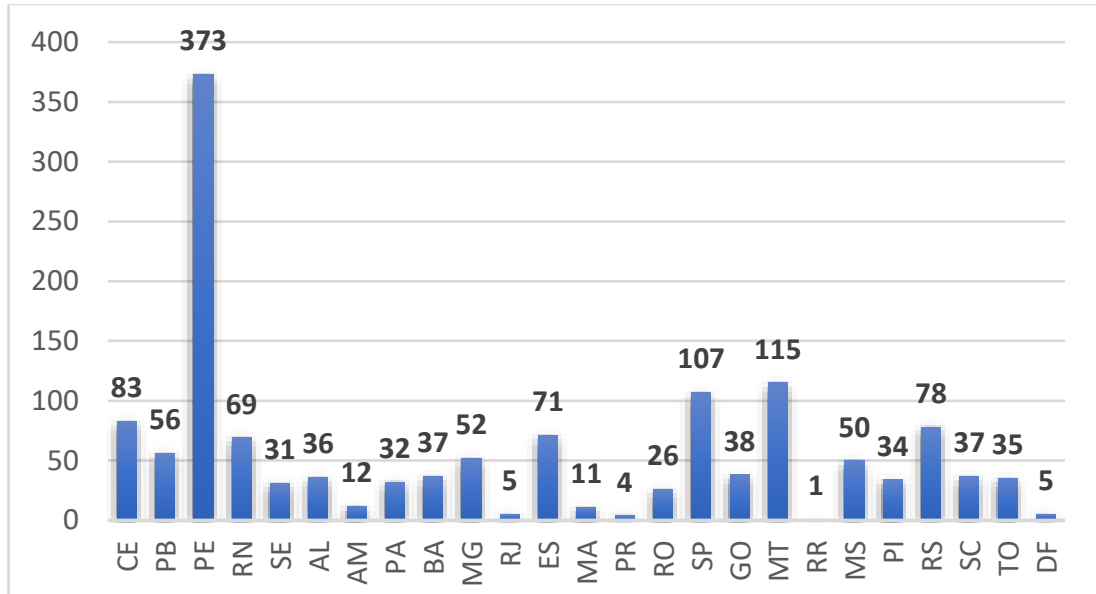

Fonte: Autores.

Destacou-se no período estudado, compreendendo o maior número de casos notificados, o estado de Pernambuco, seguido por Mato Grosso e São Paulo. Os únicos estados brasileiros que não notificaram casos da doença de 2010 a 2019 foram o Acre e Amapá.

A maioria dos casos concentraram-se nas regiões que possuem o maior efetivo de rebanho equino, desta forma, ações preventivas devem ser tomadas para evitar o avanço da doença, já que o número de susceptíveis destas regiões é elevado.

Com relação a prevalência da doença, as informações estão expressas na Figura 4.

Figura 4 - Prevalência anual/1000 cab. do Mormo nas unidades de federação de 2010 a 2019.

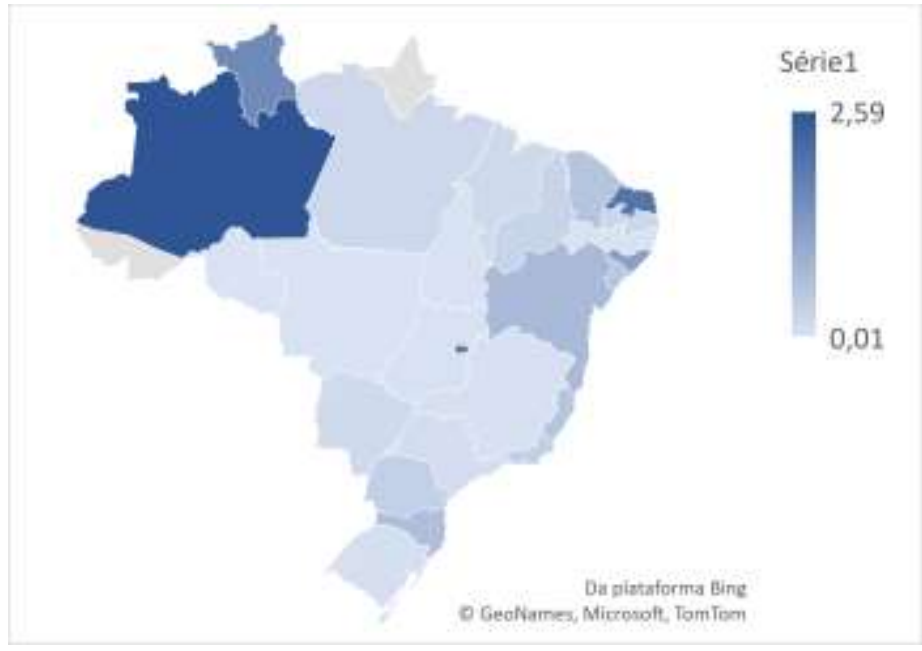

Fonte: Autores.

Os estados que apresentaram maior prevalência no período de estudo foram Amazonas (2,59/1000 cab.), Rio Grande do Norte (2,10/1000 cab.) e Distrito Federal (1,94/1000 cab.), estados estes que possuem o menor efetivo de rebanho equino. A prevalência da doença encontrada no Brasil foi de 0,25/1000 cab.

Com relação a ocorrência de focos de mormo no Brasil no período de estudo, os dados encontram-se descritos na Figura 5. 
Figura 5 - Relação entre número de casos notificados e número de focos de Mormo nas regiões brasileiras, 2010 a 2019.

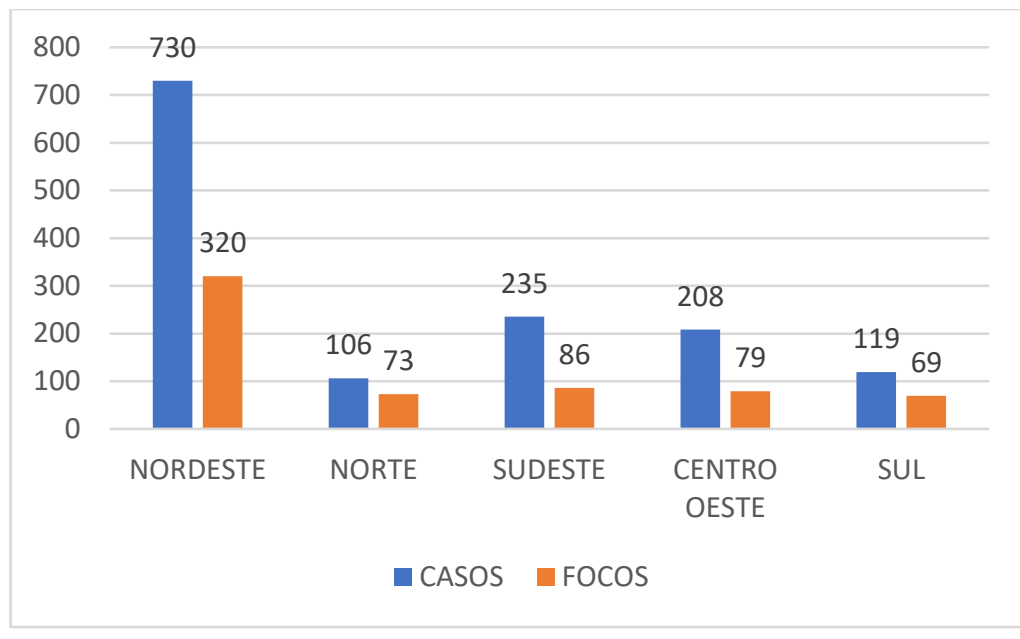

Fonte: Autores.

A região Norte apresentou menor concentração de casos por foco $(1,45)$, o que significa que a doença está mais disseminada na região, enquanto a região sudeste demonstrou maior concentração dos casos $(2,73)$ por foco. De acordo com Fonseca-Rodríguez, Pinheiro Júnior, Mota (2019), de 2005 a 2016 foram registrados 697 focos de mormo no Brasil, sendo 61,4\% na região Nordeste, corroborando com os dados apresentados nesta pesquisa.

Vários estudos vêm sendo desenvolvidos no Brasil para identificação dos casos, surtos e estudo da prevalência da doença (Machado et al., 2013; Chaves et al., 2015; Chaves et al., 2016). Devido a gravidade da afecção para a saúde humana e animal, medidas preventivas eficazes devem ser tomadas para conter o avanço da doença no Brasil.

\section{Conclusão}

Esta pesquisa permitiu concluir que o Brasil possui baixa prevalência de mormo, com concentração maior de casos e focos na região Nordeste, e maior prevalência no estado do Amazonas.

Devido a importância do mormo para a saúde pública, medidas de vigilância ativa da doença nos animais e ações de prevenção, no âmbito de Saúde Única, devem ser executadas. O investimento em programas de educação em saúde também é importante para conscientizar os proprietários e criadores de equídeos com relação a importância do controle sanitário de seu rebanho.

Desta forma estudos epidemiológicos baseados em dados oficiais e até mesmo a campo deverão ser executados com intuito de promover a saúde e prevenir a doença, com ações no âmbito da Saúde Única visando diminuir o risco de transmissão e contágio do mormo no território nacional.

\section{Agradecimentos}

Universidade Brasil, Campus Fernandópolis, São Paulo

\section{Referências}

Brasil. (2018). Instrução Normativa nº, de 16 de janeiro de 2018. Estabelece as Diretrizes Gerais para Prevenção, Controle e Erradicação do Mormo no Território Nacional. Diário Oficial da União, Brasília, p.3.

Brasil. (2008). Instrução Normativa No 17, de 8 de maio de 2008. Instituir o Programa Nacional de Sanidade dos Equídeos -PNSE, no âmbito do Ministério da Agricultura, Pecuária e Abastecimento. Diário Oficial da União, 2008. 
Carvalho, J. C. S. (2019). Frequência de casos de mormo em asininos no Brasil no período de janeiro de 2018 a abril de 2019 . Trabalho de Conclusão de Curso (Graduação em Medicina Veterinária). Universidade Federal Rural de Pernambuco, Recife.

Chaves, D. P., Brito, D. R. B., Santos, A. C. G., Vaz, J. F. R. \& Anunciação, A. R. (2015). Soroprevalência de mormo, anemia infecciosa equina e brucelose do cavalo baixadeiro. Revista Brasileira de Ciência Veterinária, 22(1), 39-42. 10.4322/rbcv.2015.317.

Chaves, N. P., Bezerra, D. C., Dos Santos Vieira, E. C., Da Silva C. \& Santos, B. C. (2016). Doenças de notificação compulsória em animais de produção no período de 2011 a 2014 no Estado do Maranhão. Revista Brasileira de Ciência Veterinária, 23(1/2), 31-36. 10.4322/rbcv.2016.026.

Coimbra, V., Falcao, M. V. D., Silva, J. G. \& Mota, R. A. (2019). Mormo: perguntas e respostas. EDUFRPE.

Dittmann, L. R., Cardoso, T. O., Romao, F. G., Barros, L. D. (2015). Aspectos clínico-patológicos do mormo em equinos - revisão de literatura. Almanaque de Medicina Veterinária e Zootecnia, 1(1), 1-5.

Fonseca-Rodríguez, O., Pinheiro Júnior, J. W. \& Mota, R. A. (2019). Spatiotemporal Analysis of Glanders in Brazil. Journal of Equine Veterinary Science, 18, 14-19. https://doi.org/10.1016/j.jevs.2019.03.216.

Galyov, E.E., Brett, P.J. \& Deshazer, D. (2010). Moleculares insights into Burkholdeira pseudomallei and Burkholdeira mallei pathogenesis. Annual Review of Microbiology, v.64, p.495-517, 2010. https://doi.org/10.1146/annurev.micro.112408.134030

IBGE. (2021). Instituto Brasileiro de Geografia e Estatística. Pesquisa da Pecuária Municipal. Disponível em: https://sidra.ibge.gov.br/Tabela/3939.

Machado, M. P. B., Silveira, P., Bandeira, J. T., Morais, R. S. M. M., Santos, F. L. \& Barçante, T. A. (2013). Prevalência de mormo no estado de Pernambuco no período de 2006 a 2011. Ciência Veterinária nos Trópicos, 16(1/2/3), 37-44.

Mota, R. A., Brito, M. F., Castro, F. J. C. \& Massa, M. (2000). Mormo em equídeos nos estados de Pernambuco e Alagoas. Pesquisa Veterinária Brasileira, 20(4),155-159. https://doi.org/10.1590/S0100-736X2000000400005.

Mota, R. A. \& Ribeiro, M. G. (2016). Mormo. In: Megid, J., Ribeiro, M. G. \& Paes, A. C. (Eds). Doenças infecciosas em animais de produção e de companhia. Roca, 423-435.

Neubauer, H., Sprague, L. D., Zacharia, R., Tomaso, H., Al Dahouk, S., Werney, R., Werney, U. \& Scholz, H. C. (2005). Serodiagnosis of Burkholderia mallei infections in horses: state-of-the-art and perspectives. Journal of Veterinary Medicine, Infectious Diseases and Veterinary Public Health, 52, 201-205. 10.1111/j.1439-0450.2005.00855.x.

Oliveira, E. C. F. (2016). Prevenção de surtos de anemia infecciosa equina e mormo nos equinos do exército brasileiro. Trabalho de Conclusão de Curso (Especialização em Ciências Militares). Escola de Aperfeiçoamento de Oficiais, Rio de Janeiro.

Pereira A. S. et al. (2018). Metodologia da pesquisa científica. UFSM.

Rosado, F. (2018). Caracterização epidemiológica do mormo em equídeos no estado da paraíba com base em dados secundários. Dissertação (Ciência Animal) - Universidade Federal da Paraíba.

Said, N. C., Nardi Junior, G. \& Domingues, P. F. (2016). Mormo em equinos e a biossegurança no agronegócio. Tekhne e Logos, 7(3).

Silveira, P., Machado, M. B., Bandeira, J., Morais, R. S. M. M., Santos F. L., Silveira, A. V. M. \& Magalhaes, C. M. B. (2013). Glanders prevalence comparision between Zona da Mata, Agreste and Sertão from Pernambuco, Brazil, from 2005 to 2011. Ciência Veterinária nos Trópicos, 16, 45-52.

Vargas, R. T., Oliveira Júnior, C. A. \& Silva, N. (2015). Situação atual do mormo no Brasil. Revista V\&Z em Minas, (127),.43-51. 